\title{
ROBUST OUTPUT REGULATION OF LINEAR SYSTEMS WITH STRUCTURAL UNCERTAINTIES
}

\author{
Minyue $\mathbf{F u}{ }^{*}$ and Jie Huang ** \\ * School of EECS, University of Newcastle, Australia \\ ** Department of Automation and Computer-Aided Engineering, \\ Chinese University of Hong Kong
}

\begin{abstract}
In this paper, we study the problem of robust regulation for a class of linear uncertain systems which admit the so-called Recursive Augmentation Structure. This structure is known to be quadratically stabilizable and is a rich class, including lowertriangular and upper-triangular structures (which correspond to the so-called backstepping and forwarding in nonlinear control) as special cases. The results of this paper provide conditions on the Recursive Augmentation Structure under which robust regulation can be achieved. Our work differs from existing work on robust regulation for linear systems in the sense that we allow large uncertainties in the system. Our work is also expected to be useful in searching for possible new structures for regulation control of nonlinear systems.Copyright (C)2005 IFAC
\end{abstract}

Keywords: Regulation Control; Robust Control; Tracking.

\section{INTRODUCTION}

Regulation control is one of the key problems in control because many control systems are designed for the purpose of tracking certain reference signals. For linear systems with a precise model, this problem was thoroughly studied in the 1970's and 1980's under the framework of internal model principle, and necessary and sufficient conditions for regulation are well understood; see, e.g., (Davison 1976) and (Francis 1977). The concept of robust regulation has been around for a long time. Robust regulation requires the existence of a fixed controller which yields regulation for all admissible uncertainties. By a simple continuity argument, it is well known (Davison (1976) and Francis (1977)) that if a "fixed" linear system is regulated by an internal model-based linear controller, the regulation property is preserved when the system parameters are slightly perturbed.

However, it is somewhat surprising that not much is known about robust regulation control for linear systems with large parametric uncertainties in view of the fact that a lot of results are available for robust stabilization of linear systems. There is no general approach for generalizing robust stabilization results to robust regulation. Although the internal model principle is still valid in the sense that robust regulation amounts to robust stabilization of the given system augmented with an internal model, the underlying technical difficulty is that the resulting robust stabilization may be difficult to solve. In general, the original system and the augmented system have different uncertainty structures.

In this paper, we study the robust regulation problem for a class of linear uncertain systems which admit the so-called Recursive Augmentation Structure. This structure was first proposed by Barmish (1982) where it was referred to as the shuffle structure. This structure was later called the antisymmetric stepwise configuration by Wei (1990) where it is proved that this structure is quadratically stabilizable via state feedback and a recursive construction algorithm is provided for stabilizing controllers. Because this structure is obtained via a sequences of the so-called up augmentations 
and down augmentations, we call it a Recursive Augmentation Structure. The structure includes the lowertriangular and upper-triangular structures (which correspond to back-stepping and forwarding in nonlinear control) as special cases.

The aim of this paper is to generalize the quadratic stabilization results of Barmish (1982) and Wei (1990) to robust regulation for the Recursive Augmentation Structure. The regulation controller employs dynamic state feedback and is based on the internal model principle. We provide two main results. The first one deals with some necessary conditions for robust regulation, whereas the second one provides a number of selections for the output which guarantees a solution to robust regulation.

\section{RECURSIVE AUGMENTATION STRUCTURE}

In this section, we introduce the Recursive Augmentation Structure initially introduced in Barmish (1982) and review the results in Wei (1990) on quadratic stabilization of this structure.

Consider the following linear time-invariant uncertain system $\Sigma(A(q), b(q))$ :

$$
\dot{x}(t)=A(q) x(t)+b(q) u(t)
$$

where $x(t) \in \mathbb{R}^{n}$ is the state, $u(t) \in \mathbb{R}$ is the control, $q \in$ $\mathbb{R}^{p}$ is the model uncertainty restricted to a prescribed bounding set $Q$.

The problem of quadratic stabilization (via linear feedback) requires finding a linear feedback controller $u(x)=k x$ for some row vector $k \in \mathbb{R}^{n}$, an $n \times n$ positive-definite symmetric matrix $P$ and constant $\alpha>$ 0 such that

$$
\begin{aligned}
L(x, q) & =\chi^{\prime}\left[A^{\prime}(q) P+P A(q)\right] x+2 \chi^{\prime} P b(q) u(x) \\
& \leq-\alpha x^{\prime} x
\end{aligned}
$$

holds for all $x \in \mathbb{R}^{n}$ and $q \in Q$. It is clear that $L(x, q)$ is the Lyapunov derivative associated with the quadratic Lyapunov function $V(x)=\chi^{\prime} P x$. The matrix $P$ is called the Lyapunov matrix, and its inverse is called the inverse Lyapunov matrix.

The class of uncertain systems $\Sigma(A(q), b(q))$ studied in (Wei 1990) admits the so-called standard form with structural independent uncertainties (or standard form for short), which is defined below:

Definition 1. Take the $(n+1) \times(n+1)$ matrix

$$
M(q)=\left\{m_{i j}(q)\right\}=\left[\begin{array}{cc}
A(q) & b(q) \\
0 & 0
\end{array}\right]
$$

Then, the uncertain system $\Sigma(A(q), b(q))$ is said to be in the standard form if for every entry $m_{i j}(q)$, the following properties hold:

- If it is a superdiagonal entry, i.e., $j=i+1$, then, it is an uncertain term which varies independently in $\left[\underline{r}_{i j}, \bar{r}_{i j}\right]$, where either $\underline{r}_{i j}>0$ or $\bar{r}_{i j}<0$, i.e., $m_{i j}$ is bounded and bounded away from zero. For notational simplicity, these entries will be denoted by $\theta$.

- If it is a non-superdiagonal entry, i.e., $j \neq i+$ 1 , then it is either zero or an uncertain term which varies independently in $\left[\underline{r}_{i j}, \bar{r}_{i j}\right]$ for any $\underline{r}_{i j}$ and $\bar{r}_{i j}$, i.e., $\left|m_{i j}\right|$ is either zero all the time or varies in an arbitrarily large but bounded region. Similarly, these entries, if not identically zero, will be denoted by $*$.

Definition 2. An uncertain system $\Sigma(A(q), b(q))$ in the standard form is said to admit an Recursive Augmentation Structure if its corresponding matrix $M(q)$ as in (3) satisfies the following condition: If $p \geq k+2$ and $m_{k p}(q) \not \equiv 0$, then $m_{u v}(q) \equiv 0$ for all $u \geq v, u \leq p-$ 1 and $v \leq k+1$.

The following key result is cited from (Wei 1990):

Lemma 3. Suppose $\Sigma(A(q), b(q))$ is in the standard form. Then, it is quadratically stabilizable if and only if it admits the Recursive Augmentation Structure.

Definition 4. Let $\Sigma(A(q), b(q))$ be $n$-dimensional. A down-augmented system $\Sigma^{+}\left(A^{+}(q), b^{+}(q)\right)$ is given by

$$
A^{+}(q)=\left[\begin{array}{cc}
A(q) & b(q) \\
* * \cdots * & *
\end{array}\right] ; b^{+}(q)=\left[\begin{array}{l}
0 \\
\theta
\end{array}\right] \in \mathbb{R}^{n+1},
$$

where $*$ and $\theta$ are uncertain entries as defined earlier. Similarly, suppose $\Sigma(A(q), b(q))$ is in the following form:

$$
A(q)=\left[\begin{array}{cc}
0 & A^{-}(q) \\
* * * \cdots *
\end{array}\right] ; \quad b(q)=\left[\begin{array}{l}
0 \\
\theta
\end{array}\right]
$$

for some $(n-1) \times(n-1)$ uncertain matrix $A^{-}(q)$ with $n \geq 1$. An up-augmented system $\Sigma^{+}\left(A^{+}(q), b^{+}(q)\right)$ is given by

$$
A^{+}(q)=\left[\begin{array}{ccc}
0 & \theta & * * \cdots * \\
0 & 0 & A^{-}(q) \\
* & * * * * \cdots *
\end{array}\right] ; b^{+}(q)=\left[\begin{array}{c}
0 \\
b(q)
\end{array}\right]
$$

The system $\Sigma(A(q), b(q))$ will be referred to as the generating system.

The Recursive Augmentation Structure is constructed via a sequence of up and down augmentations, originated from a scalar system

$$
\dot{x}_{k}=a_{k} x_{k}+\theta u
$$

where $a_{k}$ is either 0 or $*$. The system (7) will be referred to as the generating system.

\section{REVIEW OF LINEAR REGULATION}

Research on regulation problems was very active in the 1970's; see, for example, Davison (1976) and 
Francis (1977) and their bibliographies. The main results that are pertinent to our paper are summarized below.

Consider an uncertain LTI system

$$
\begin{aligned}
& \dot{x}(t)=(A+\delta A) x+(b+\delta b) u+(E+\delta E) v \\
& e(t)=(c+\delta c) x+(d+\delta d) u+(F+\delta F) v
\end{aligned}
$$

where $x \in \mathbb{R}^{n}$ and $u \in \mathbb{R}$ are as before, $v \in \mathbb{R}^{m}$ is the reference signal, $e \in \mathbb{R}$ is the regulation error, $A, b, c, d, E$ and $F$ are given, and the $\delta$ terms represent perturbations with arbitrarily small sizes. Assume that the reference signal is generated by

$$
\dot{v}=A_{1} v
$$

where $A_{1}$ is a known matrix with eigenvalues in the closed right-half plane. The reference model is driven by some nonzero initial value $v(0)$.

The regulation problem is to design a (fixed) linear feedback control law such that the closed-loop system is asymptotically stable and the regulation error approaches zero asymptotically, regardless the perturbations $\delta A, \delta b$, etc., provided they are sufficiently small. In this paper, we consider dynamic state feedback defined by

$$
\begin{aligned}
\dot{z} & =G_{1} z+G_{2} e \\
u & =K_{1} x+K_{2} z
\end{aligned}
$$

The well-known internal model principle below asserts that the regulation problem is essentially a stabilization problem.

Lemma 5. (Francis (1977) and Davison (1976)) Given the system in (8) and reference model in (9), assume, without loss of generality, that $(A, b)$ is stabilizable. Then,

- The necessary and sufficient condition for solving the dynamic state feedback regulation problem is that the eigenvalues of $A_{1}, \sigma\left(A_{1}\right)$, do not overlap with the zeros of system $\Sigma(A, b, c, d)$.

- Under the condition above, then a regulating controller (10) contains an internal model of the reference signal, i.e., $\sigma\left(A_{1}\right) \subset \sigma\left(G_{1}\right)$ and $\left(G_{1}, G_{2}\right)$ is a controllable pair. Further, with any internal model, the controller regulates the system if and only if it stabilizes the system.

\section{ROBUST REGULATION}

The robust regulation problem is very similar to the regulation problem except that the model uncertainties $(\delta A, \delta b$, etc.) are now allowed to be large. For this reason, we rewrite (8) as

$$
\begin{aligned}
& \dot{x}(t)=A(q) x+b(q) u+E(q) v \\
& e(t)=c(q) x+d(q) u+F(q) v
\end{aligned}
$$

where $q \in Q$ representing constant uncertain parameters, $A(\cdot), b(\cdot), c(q), d(q), E(q)$ and $F(q)$ are continuous functions of $q$, and $Q$ is a given compact set called the bounding set. The controller takes the form of (10). In particular, it does not depend on $q$ but it may depend on $Q$. The robust regulation problem requires stabilization and regulation to be achieved for all $q \in Q$. In this paper, we only consider dynamic state feedback (10).

Recall that stabilizability is a necessary condition for regulation. It is natural for robust regulation that we only consider uncertain systems which are robustly stabilizable and are so via state feedback because (10) is the required control law. Motivated by the above, we study uncertain systems (11) which satisfy the following conditions:

i) $(A(q), b(q))$ admits the Recursive Augmentation Structure;

ii)

for some $k$, where

$$
e=c_{k}(q) x+F(q) v
$$

$$
c_{k}(q)=[* * \cdots * \theta * \cdots *]
$$

with $\theta$ being at the $k$ th entry.

iii) The pair $\left(A_{1}, F(q)\right)$ is detectable for all $q \in Q$ and $A_{1}$ has all the eigenvalues in the closed right-half plane.

Without loss of generality, we assume that $A_{1}$ is in the following form:

$$
A_{1}=\left[\begin{array}{ccccc}
0 & 1 & 0 & \ldots & 0 \\
0 & 0 & 1 & 0 & \vdots \\
\vdots & & & \ddots & 0 \\
0 & 0 & \ldots & 0 & 1 \\
\alpha_{1} & \alpha_{2} & \ldots & \alpha_{m-1} & \alpha_{m}
\end{array}\right] .
$$

Accordingly, we choose the internal model $\left(G_{1}, G_{2}\right)$ in (10) to be in the controllable canonical form:

$$
\begin{aligned}
\dot{z}_{1} & =z_{2} \\
& \cdots \\
\dot{z}_{m-1} & =z_{m} \\
\dot{z}_{m} & =\alpha_{1} z_{1}+\cdots+\alpha_{m} z_{m}+e
\end{aligned}
$$

Theorem 6. Suppose the regulation error $e(t)$ takes the form (12) and that $(A(q), b(q))$ admits the Recursive Augmentation Structure. Then, the following results hold for dynamic state feedback robust regulation:

- If $k>1$, then a necessary condition for robust regulation is $\alpha_{1} \neq 0$, even when $c_{k}(q)=$

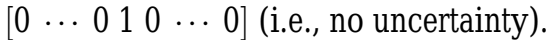

- Robust regulation can be achieved if $k=1$ and $\alpha_{i}=0$ for all $i=1, \cdots, m$.

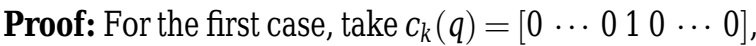
i.e., $e=x_{k}+F(q) v$ for $k>1$. If $\alpha_{1}=0$, we choose a special reference signal: $v_{1}=\beta ; v_{2}=0, \cdots, v_{m}=0$, where $\beta$ is a nonzero constant. Certainly, this signal fits the reference model because $\alpha_{1}=0$. Since $\left(A_{1}, F(q)\right)$ is detectable, $F(q) \vee$ must also be a nonzero constant. Take

$$
\dot{x}_{k-1}=\theta x_{k}
$$


i.e., set possible $*$ terms to zero. Suppose on the contrary that robust regulation is achieved. Then, $x_{k}$ should approach to $-F(q) v$ asymptotically, i.e., a nonzero constant. This implies that $x_{k-1}$ would have to approach to infinity asymptotically. Thus, we have unbounded state for bounded input, i.e., the system is not stable. By this contradiction, we conclude that the system cannot be robustly regulated.

For the second case, we simply amalgamate the internal model (15) with (11). By setting $v=0$, we obtain

$$
\begin{aligned}
\dot{z}_{1} & =z_{2} \\
& \cdots \\
\dot{z}_{m-1} & =z_{m} \\
\dot{z}_{m} & =\theta x_{1}+* x_{2}+\cdots+* x_{n} \\
\dot{x} & =A(q) x+b(q) u
\end{aligned}
$$

It is clear that the system above is obtained by upaugmentations from $(A(q), b(q))$, so it admits the Recursive Augmentation Structure because $(A(q), b(q))$ does so. Hence, it is robustly stabilizable by $u=K_{1} \chi+$ $K_{2} z$. By the internal model principle, this controller also robustly regulates the system (11).

Theorem 7. Suppose the regulation error $e(t)$ takes the form (12) and that $(A(q), b(q))$ admits the Recursive Augmentation Structure generated from the generating system (7), i.e., the regulated state variable is the variable of the generating system for the Recursive Augmentation Structure. Then, the following results hold for dynamic state feedback robust regulation:

(i) Robust regulation can be achieved if $k=1$ (i.e., $(A(q), b(q))$ involves down-augmentations only) and $c_{k}(q)=\left[\begin{array}{llll}\theta & 0 & \cdots & 0\end{array}\right]$.

(ii) Robust regulation can be achieved if $k>1$,

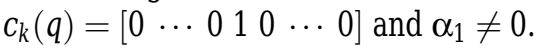

(iii) As a special (but interesting) case of (ii) above, robust regulation can be achieved if $\alpha_{1} \neq 0, k=$ $n-1$ with $c_{k}(q)=\left[\begin{array}{lllll}0 & \cdots & 0 & 1 & 0\end{array}\right]$ or $k=n$ with $c_{k}(q)=\left[\begin{array}{llll}0 & \cdots & 0 & 1\end{array}\right]$, and the system $(A(q), b(q))$ involves up-augmentations only, i.e.,

$$
\begin{aligned}
\dot{x}_{1} & =\theta x_{2}+* x_{3}+\cdots+* x_{n} \\
& \cdots \\
\dot{x}_{n-2} & =\theta x_{n-1}+* x_{n} \\
\dot{x}_{n-1} & =\theta x_{n} \\
\dot{x}_{n} & =* x_{1}+\cdots+* x_{n}+\theta u
\end{aligned}
$$

Proof: All we need to show is that in either case above, the amalgamated system, consisting of (11) and (15) with $v=0$, admits the Recursive Augmentation Structure. The first case is easy to check because the amalgamated system (with $v=0$ ) is given by

$$
\begin{aligned}
\dot{z}_{1} & =z_{2} \\
& \cdots \\
\dot{z}_{m-1} & =z_{m} \\
\dot{z}_{m} & =\alpha_{1} z_{1}+\cdots+\alpha_{m} z_{m}+\theta x_{1} \\
\dot{x}_{1} & =\theta x_{2} \\
& \cdots \\
\dot{x}_{n-1} & =* x_{1}+\cdots * x_{n}+\theta u
\end{aligned}
$$

It can be verified that the above admits the Recursive Augmentation Structure. Therefore, there exists a robust stabilizing controller $u=K_{1} x+K_{2} z$. According to the internal model principle, this controller will also robustly regulates the system.

For the second case, we combine the generating system (7) and the first series of down-augmentations (if any) before up-augmentations as follows:

$$
\begin{aligned}
\dot{x}_{k} & =\theta x_{k+1} \\
& \ldots \\
\dot{x}_{k+j-1} & =* x_{k+1}+\cdots * x_{k+j-1}+\theta x_{k+j} \\
\dot{x}_{k+j} & =* x_{k}+\cdots * x_{k+j}+\theta u
\end{aligned}
$$

for $j \geq 0$. Note that if $j=0$, then down-augmentations are void, i.e., augmented system $(A(q), b(q))$ starts with up-augmentations. Also note that in the first $j$ lines above, the $x_{k}$ term does not appear on the right hand side. This is a requirement for the subsequent up-augmentation(s); see (6).

Define

$$
\bar{x}_{k}=\alpha_{1} z_{1}+x_{k}
$$

Combining (20) with (15) and replacing $x_{k}$ by $\bar{x}_{k}$ gives the following amalgamated system:

$$
\begin{array}{ll}
\dot{z}_{1} & =z_{2} \\
& \cdots \\
\dot{z}_{m-1} & =z_{m} \\
\dot{z}_{m} & =\alpha_{2} z_{2}+\cdots+\alpha_{m} z_{m}+\bar{x}_{k} \\
\dot{\bar{x}}_{k} & =\theta \alpha_{1} z_{2}+\theta x_{k+1} \\
\dot{x}_{k+1} & =* x_{k+1}+\theta x_{k+2} \\
& \cdots \\
\dot{x}_{k+j-1} & =* x_{k+1}+\cdots+* x_{k+j-1}+\theta x_{k+j} \\
\dot{x}_{k+j} & =*\left(-\alpha_{1} z_{1}+\bar{x}_{k}\right)+\cdots+* x_{k+j}+\theta u
\end{array}
$$

Note that in the 5th line above, the term $\alpha_{1} z_{2}$ should be replaced by $\alpha_{1} \bar{x}_{k}$ if $m=1$. Anyway, the structure above involves only down-augmentations.

Now consider the next series of up-augmentations

$$
\begin{aligned}
\dot{x}_{k-i} & =\theta x_{k-i+1}+* x_{k-i+2}+\cdots+* x_{k+j} \\
& \ldots \\
\dot{x}_{k-1} & =\theta x_{k}+* x_{k+1}+\cdots+* x_{k+j}
\end{aligned}
$$

for some $i \geq 1$. We have

$$
\begin{aligned}
\dot{x}_{k-i}= & \theta x_{k-i+1}+* x_{k-i+2}+\cdots \\
& +*\left(-\alpha_{1} z_{1}+\bar{x}_{k}\right)+\cdots+* x_{k+j} \\
& \ldots \\
\dot{x}_{k-1}= & -\alpha_{1} \theta z_{1}+\theta \bar{x}_{k}
\end{aligned}
$$

Stacking them on top of (22), the amalgamated system also admits the Recursive Augmentation Structure. This process can continue until all state variables $x_{1}, \cdots, x_{n}$ are exhausted. Hence, the Recursive Augmentation Structure is preserved, and like the first case, robust regulation is achieved by some $u=K_{1} x+$ $K_{2} z$.

The third case is a special case of (ii) because the system (13) can be viewed as augmented from either $x_{n}$ with $n$ up-augmentations or $x_{n-1}$ through a down-augmentation first followed by $n-1$ upaugmentations. 
Remark. We point out that two results in Theorem 7 have counterparts in nonlinear regulation. These are Case (i), which can be found in Chen and Huang (2002) and Isidori (1997), and Case (iii) with $k=n$, which can be found in Marcoli, Isidori and Serrani (2001). Roughly speaking, the nonlinear counterparts of these results allow the $*$ and $\theta$ terms to be replaced by nonlinear functions (but with some restrictions); see the references above for details. However, no nonlinear counterparts exist so far for the more general results in Theorem 7.

\section{CONTROLLER DESIGN AND ILLUSTRATIVE EXAMPLE}

The results given in Theorem 7 address the solvability of robust regulation. This section discusses the controller design issue. We will introduce a design algorithm and demonstrate it through an example.

Recall that we reduce a robust regulation problem to a robust stabilization problem via the internal model principle and that the Recursive Augmentation Structure is preserved in the process. Hence, it is sufficient to explain how to design a quadratic stabilizer for an Recursive Augmentation Structure. This is done using the three results in the theorem below. The first two results are from Barmish (1985) and the third one from Wei (1990).

Theorem 8. Given an $n$-th order uncertain system $\Sigma=$ $(A(q), b(q))$ with $b(q)=\left[\begin{array}{lllll}0 & 0 & \ldots & 0 & \theta\end{array}\right]^{T}$, continuous $A(q)$ and a compact bounding set $Q$, it is quadratically stabilizable if and only if

$$
b_{\perp}\left(S A^{T}(q)+A(q) S\right) b_{\perp}^{T}<0, \quad \forall q \in Q,
$$

where $b_{\perp}=\left[\begin{array}{ll}I_{n-1} & 0\end{array}\right]$. If the condition above holds, then a stabilizing controller is given by

$$
k=-\gamma b_{0}^{T} S^{-1},
$$

where $b_{0}=\left[\begin{array}{lllll}0 & 0 & \ldots & 0 & 1\end{array}\right]^{T}$ and $\gamma$ is any scalar satisfying

$$
\left[\begin{array}{cc}
b_{\perp} \Omega(q) b_{\perp}^{T} & b_{\perp} \Omega(q) b_{0}^{T} \\
b_{0}^{T} \Omega(q) b_{\perp}^{T} & b_{0}^{T} \Omega(q) b_{0}^{T}-\gamma \theta
\end{array}\right]<0, \quad \forall q \in Q,
$$

where $\Omega(q)=S A^{T}(q)+A(q) S$.

Suppose $\Sigma$ above is is quadratically stabilizable, $S=$ $S^{T}>0$ is the corresponding inverse Lyapunov matrix and $k$ is the corresponding controller gain. Then, any down augmented system $\Sigma^{+}=\left(A^{+}(q), b^{+}(q)\right)$ is quadratically stabilizable via the following inverse Lyapunov matrix

$$
S^{+}=\left[\begin{array}{cc}
S & S k^{T} \\
k S & S_{n+1}+k S k^{T}
\end{array}\right],
$$

where $s_{n+1}$ is any positive number. The corresponding controller gain $k^{+}$can be found using the first part of this theorem.

Suppose $\Sigma$ above is is quadratically stabilizable, $S=$ $S^{T}>0$ is the corresponding inverse Lyapunov matrix.
Then, any up augmented system $\Sigma^{+}=\left(A^{+}(q), b^{+}(q)\right)$ is quadratically stabilizable via the following inverse Lyapunov matrix

$$
S^{+}=\left[\begin{array}{cc}
S_{0}+\gamma^{2} c_{0}^{T} S^{-1} c_{0} & -\gamma c_{0}^{T} \\
-\gamma c_{0} & S
\end{array}\right],
$$

where $c_{0}=\left[\begin{array}{lllll}1 & 0 & 0 & \ldots & 0\end{array}\right]^{T}, s_{0}$ is any positive number, and $\gamma$ is chosen to satisfy

$$
b_{\perp}^{+}\left(S^{+}\left(A^{+}(q)\right)^{T}+A^{+}(q) S^{+}\right)\left(b^{+}\right)_{\perp}^{T}<0, \forall q \in Q,
$$

where $b_{\perp}^{+}=\left[\begin{array}{ll}I_{n} & 0\end{array}\right]$.

Controller Design Algorithm: We assume that the conditions in Theomrem 7 are satisfied.

Step 1: Form the amalgamated system $\bar{\Sigma}$ as in (22) which consists of an internal model of the reference system with the given uncertain system $\Sigma$. Initialize $\Sigma_{0}$ to be the generating system for $\Sigma$, choose the corresponding inverse Lyapunov matrix $S_{0}=$ 1 and select the corresponding controller gain $k_{0}$ that (quadratically) stabilizes the generating system. Denote by $\Sigma_{i}, i=1,2, \cdots, \bar{n}$, the sequence of augmented systems obtained recursively from $\Sigma_{0}$ which leads to the final amalgamated system, i.e., $\Sigma_{\bar{n}}=\dot{\Sigma}$.

Step 2: For $i=1,2, \ldots, \bar{n}$, design the inverse Lyapunov matrix $S_{i}$ for the augmented system $\Sigma_{i}$ using Theorem 8. Then, set $\bar{S}=S_{\bar{n}}$.

Step 3: Design a quadratic stabilizer for $\bar{\Sigma}$ using Theorem 8.

Illustrative Example: The uncertain system $\Sigma$ in this example is given by

$$
\begin{aligned}
& \dot{x}_{1}=x_{2}+\alpha_{2} x_{3} \\
& \dot{x}_{2}=\theta_{1} x_{3} \\
& \dot{x}_{3}=\alpha_{1} x_{1}+\theta_{2} u \\
& y=x_{2},
\end{aligned}
$$

where $\alpha_{0} \in[-0.2,0.2]$ and $\theta_{i} \in[0.8,1.2]$ for $i=1,2$. The output $y$ is required to track a sinusoidal signal $w$ generated by

$$
\begin{aligned}
& \dot{v}_{1}=v_{2} \\
& \dot{v}_{2}=-v_{1} \\
& w=g v_{1}
\end{aligned}
$$

with $g$ to be specified. It is verified that $\Sigma$ is a Recursive Augmentation Structure with the generating system

$$
\dot{x}_{2}=\theta_{1} u
$$

and the sequence of augmentations being down-up. This falls into Case (iii) of Theorem 7, therefore robust regulation can be achieved. The amalgamated system is given by

$$
\begin{aligned}
& \dot{x}_{1}=x_{2}+\alpha_{2} x_{3} \\
& \dot{z}_{1}=z_{2} \\
& \dot{z}_{2}=-z_{1}+x_{2}-g(t) v_{1} \\
& \dot{x}_{2}=\theta_{1} x_{3} \\
& \dot{x}_{3}=\alpha_{1} x_{1}+\theta_{2} u
\end{aligned}
$$

Following Step 1 in the Controller Design Procedure, defining $\bar{x}_{2}=-z_{1}+x_{2}$, we transform the system above into $\bar{\Sigma}$ below: 


$$
\begin{aligned}
& \dot{x}_{1}=z_{1}+\bar{x}_{2}+\alpha_{2} x_{3} \\
& \dot{z}_{1}=z_{2} \\
& \dot{z}_{2}=\bar{x}_{2}-g(t) v_{1} \\
& \dot{\bar{x}}_{2}=-z_{2}+\theta_{1} x_{3} \\
& \dot{x}_{3}=\alpha_{1} x_{1}+\theta_{2} u,
\end{aligned}
$$

which we need to stabilize quadratically (when $v_{1}$ is set to zero). It is easy to verify that the system above admits the Recursive Augmentation Structure and the sequence of augmentations is down-down-down-up with the generating system being

$$
\dot{z}_{1}=z_{2},
$$

which is easily stabilized with any controller gain $k_{0}<0$ and inverse Lyapunov matrix (scalar in this case) $S_{0}>0$. Next, we follow Step 2 in the Controller Design Algorithm obtain the inverse Lyapunov matrix $\bar{S}$ for $\bar{\Sigma}$. The result is given by

$$
\bar{S}=\left[\begin{array}{ccccc}
264.69 & -10.0 & 0 & 0 & 0 \\
-10.0 & 4.11 & -0.5 & -1.04 & -1.0 \\
0 & -0.5 & 1.04 & -0.5 & -2.0 \\
0 & -1.04 & -0.5 & 1.1 & -3.0 \\
0 & -1.0 & -2.0 & -3.0 & 74.08
\end{array}\right]
$$

The relevant design parameters for each recursion (i.e., $\gamma, s_{0}$ or $s_{n+1}$ as specified in Theorem 8) can be identified from $\bar{S}$. Finally, we follow Step 3 in the design procedure to derive the following controller gain:

$k=[-1.377-11.018-16.518-12.513-5.809]$.

Figure 1 shows a simulation of the system. The initial conditions of $\Sigma$ are set to zero, but for the reference, $v(0)=\left[\begin{array}{ll}1 & 0\end{array}\right]$ is used. The two trajectories correspond to the reference signal and the output of the system. For $0 \leq t \leq 20$, the magnitude of the reference signal $g=1$. For $t>20, g=-0.5$ is used. The uncertain parameters are taken to be

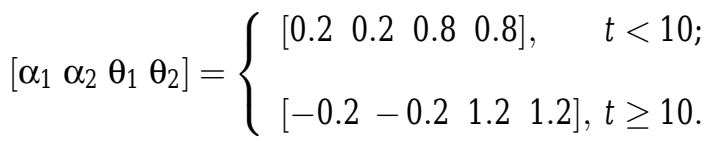

We see that the output converges well in 5 seconds or so after each parameter change.

\section{CONCLUSIONS}

In this paper, we have derived a number of results on the problem of robust regulation for linear uncertain systems. These uncertain systems are very general and admit the Recursive Augmentation Structure. On one hand, our results can be viewed as generalizations of those in Barmish (1982) and Wei (1990) on quadratic stabilization to robust regulation. On the other hand, the results given in this paper can be used to motivate new structures for nonlinear regulation. It is likely that nonlinear systems which admit a generalized Recursive Augmentation Structure (with the $*$ and $\theta$ terms

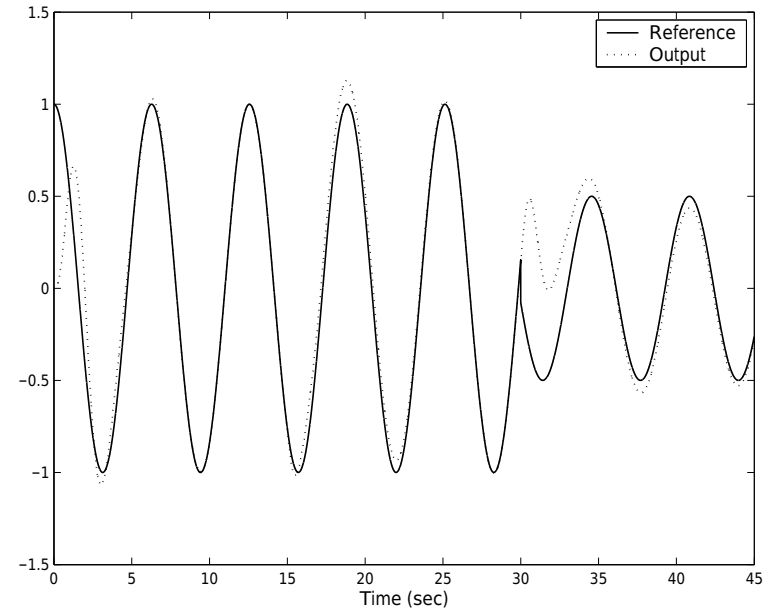

Fig. 1. Simulation of the Illustrative Example

replaced by appropriate nonlinear functions) and similar constraints on the regulated output as in Theorems 6 and 7 can also have solutions to robust regulation problems. This is a subject to be further studied.

\section{REFERENCES}

B. R. Barmish (1982). On enlarging the class of stabilizable linear systems with uncertainty. Proc. IEEE Asilomar Conf. Comput., Circuits Syst., Monterey, CA.

B. R. Barmish (1985). Necessary and sufficient conditions for quadratic stabilizability of an uncertain system. J. Opt. Theory Appl., 46, 399-408.

Z. Y. Chen and J. Huang (2002). Global output regulation for lower triangular nonlinear systems. Proceedings of the $15^{\text {th }}$ IFAC world congress, Barcelona, Spain.

E. J. Davison (1976). The robust control of a servomechanism problem for linear time-invariant multivariable systems. IEEE Trans. Auto. Contr., AC-21, 25-34.

B. Francis (1977). The linear multivariable regulator problem. SIAM J. Contr. Opt., 15, 486-505.

A. Isidori (1997). A Remark on the problem of semiglobal nonlinear output regulation. IEEE Trans. Auto. Contr., 42, 1734-1738.

L. Marcoli, A. Isidori and A. Serrani (2001). Global input disturbance suppression for a class of uncertain nonlinear systems. Proc. IFAC Symp. Nonlinear Contr. Design, 668-672.

K. Wei (1990). Quadratic stabilizability of linear systems with structural independent time-varying uncertainties. IEEE Trans. Auto. Contr., 35, 268277. 